\title{
A Hybrid Forecasting Model for Nonstationary and Nonlinear Time Series in the Stochastic Process of $\mathrm{CO}_{2}$ Emission Trading Price Fluctuation
}

\author{
Shanglei Chai, ${ }^{1}$ Mo Du ${ }^{1},{ }^{2}$ Xi Chen, ${ }^{3}$ and Wenjun Chu ${ }^{1}$ \\ ${ }^{1}$ School of Business, Shandong Normal University, 88 Wenhua Road, Jinan 250014, China \\ ${ }^{2}$ School of Accounting, Shandong Youth University of Political Science, 31699 East Jingshi Road, Licheng District, Jinan 250103, \\ China \\ ${ }^{3}$ Department of Management Engineering, School of Economics \&Management, Xidian University, Xi'an 710071, China
}

Correspondence should be addressed to Mo Du; dumo1977@163.com

Received 16 June 2020; Accepted 16 July 2020; Published 4 August 2020

Guest Editor: Wenguang Yu

Copyright (C) 2020 Shanglei Chai et al. This is an open access article distributed under the Creative Commons Attribution License, which permits unrestricted use, distribution, and reproduction in any medium, provided the original work is properly cited.

Predicting $\mathrm{CO}_{2}$ emission prices is an important and challenging task for policy makers and market participants, as carbon prices follow a stochastic process of complex time series with nonstationary and nonlinear characteristics. Existing literature has focused on highly precise point forecasting, but it cannot correctly solve the uncertainties related to carbon price datasets in most cases. This study aims to develop a hybrid forecasting model to estimate in advance the maximum or minimum loss in the stochastic process of $\mathrm{CO}_{2}$ emission trading price fluctuation. This model can granulate raw data into fuzzy-information granular components with minimum (Low), average $(R)$, and maximum (Up) values as changing space-description parameters. Furthermore, it can forecast carbon prices' changing space with Low, $R$, and Up as inputs to support a vector regression. This method's feasibility and effectiveness is examined using empirical experiments on European Union allowances' spot and futures prices under the European Union's Emissions Trading Scheme. The proposed FIG-SVM model exhibits fewer errors and superior performance than ARIMA, ARFIMA, and Markov-switching methods. This study provides several important implications for investors and risk managers involved in trading carbon financial products.

\section{Introduction}

Since the launch of the European Union's Emissions Trading Scheme (EU ETS), carbon dioxide $\left(\mathrm{CO}_{2}\right)$ emission certificates have been cultivated as a scarce resource. Furthermore, EU allowances (EUAs) have been actively traded in spot and its derivative future markets as a new class of financial assets. Consequently, reinforced carbon price signals coupled with complementary policies can effectively mitigate the costs of global carbon emissions. With carbon markets' rapid growth, carbon prices have become more volatile due to the influence of many potential fundamentals, which are drivers that have led to drastic changes in carbon prices. Irregular and unexpected price fluctuations have increased the risk in the carbon market, which affects participants' confidence and emission-mitigation targets [1]. Therefore, it is essential to understand carbon prices' dynamics and establish a scientific carbon price-forecasting model, as predicting carbon prices is useful for reducing market risk [2]. This study aims to predict EUA prices in an effort to not only assist entities regulated under the EU ETS in managing risk but also benefit policy makers concerned with this pricing mechanism.

Meanwhile, a scientific prediction tool in the investment field can help speculators seek short-term arbitrage opportunities from volatility trades and develop efficient investment strategies in the carbon market. Given the significance of scientific prediction models, many studies have presented several methods to forecast carbon prices and this literature is commonly divided into two strands [3]. First, the literature forecasts carbon prices in the context of classic statistical methods, including the ARIMA, GARCH, 
VAR, and Markov-switching models [4-7]. These typically manifest as a parameterisation or linearity, and most of their estimation processes use the maximum likelihood method. In this case, the time-series variables must obey an assumption of normal distribution or the sample observation must be sufficiently long. However, carbon prices in practice do not possess linear characteristics or may not be generally predictable in a linear manner [8-10]. Chevallier [9] detects the strong nonlinearities existing in carbon prices' fluctuations to indicate that a nonparametric forecasting model can substantially reduce prediction errors. Arouri et al. [8] capture the asymmetry and nonlinearity of carbon prices and confirm the necessity of establishing a nonlinear carbon price-prediction model. To achieve a better nonlinear approximation, empirical studies on carbon prices' forecasting have increasingly built a second strand of the literature using computational intelligence techniques [3,11-18]. These primarily refer to the artificial neural network, fuzzy logic, evolutionary algorithm, support vector machine (SVM), and hybrid models, which exploit the advantages of the intelligence algorithm and statistical models. Some early studies preferred to use the neural network for carbon price forecasting, with favourable results $[11,12]$. Although the neural network exhibits a good nonlinear approximation ability, it is prone to fall into local optimum, overtraining and undertraining, and has poor generalisability. Therefore, the neural network exhibits certain limitations in applying a replicated time series.

Support vector machines (SVM) as proposed by Cortes and Vapnik [19] differ from traditional learning theory, in which SVM obeys the principle of structural rather than empirical risk minimisation, and thus, it exhibits good generalisability. Given this advantage, this method has been used in carbon prices forecasting issues to demonstrate a higher robustness than other methods $[14,15,17,18]$. A common, noteworthy feature of these papers is that a variety of hybrid models are constructed using SVM and such traditional time-series analysis methods as ARIMA, GARCH, and empirical mode decomposition, among others. The empirical results suggest that a hybrid model performs well in nonstationary and nonlinear time-series analyses, and especially in forecasting carbon prices. Unlike the previously mentioned studies which primarily predict the EU carbon market, some studies have predicted the emerging carbon market in China $[3,13,20]$. Despite the different topics investigated, these papers reach the same conclusion, in which hybrid models have a higher forecasting accuracy than benchmark models.

Although many have studied the previously mentioned time-series models for point forecasting with high precision in numerical calculations, these may not be absolutely essential for relevant real-world information users. For example, it is more suitable for stock investors to predict the changing space for future stock prices and thus make profits from them, rather than providing some specific numbers for stock price. This is because the fluctuations in stock price forecasting help investors make reasonable decisions to seize arbitrage opportunities. The same principle applies to the carbon market, as it is similar to the stock market. Thus, precision is not an absolutely necessary condition for human thinking or reasoning, so the range of trusted variation of time series is satisfactory for some decision makers [21]. In addition, previous studies on carbon price forecasting mainly focus on point forecasting, but point forecasting in most cases cannot correctly solve the uncertainties related to carbon price datasets [22]. On the contrary, changing space predictions can quantify the influence of uncertain factors on the results so that the original value is within the prediction interval determined by the upper and lower limits of the interval. Therefore, it is highly significant for most investors to make changing space predictions of carbon prices in practical application rather than point forecasting, the latter of which has highly precise numerical values.

Consequently, this study aims to develop a novel carbon prices' changing space forecasting model to make up for previous point forecasting methods. It compensates for a lack of point forecasting by using the original data that must be processed through fuzzy information granulation (FIG) as first proposed by Zadeh [23]. This useful tool provides appropriate solutions in developing a fast-space forecasting model for complex time-series process with nonstationary and nonlinear characteristics [21]. Furthermore, studies increasingly use information granulation to process complicated time-series data to improve prediction accuracy [24-28]. Each information granule represents the fuzziness and uncertainty of a certain period. The granulated data can be analysed after the original data is preprocessed using FIG and then forecasts can be conducted.

We consider this motivation in developing fast-changing space predictors-and not point predictions-using a FIGSVM model in this paper, with the following primary contributions to existing studies. First, the input scales for SVM can be reduced by FIG and therefore forecasting efficiency may be improved. This method granulates timeseries carbon prices into Low, $R$, and Up categories to rapidly predict the possible minimum, average, and maximum of complicated time-series. Second, our proposed FIG-SVM model can forecast carbon prices' changing space using a novel framework with minor errors and good generalisability. The experimental results demonstrate its superiority to the other benchmark models, such as the ARIMA, ARFIMA, and Markov-switching models. Third, a credible approach to carbon prices' changing space forecasting is more helpful to some decision makers in carbon markets rather than the point-forecasting method commonly used in the existing literature. This provides several important implications for speculators to catch arbitrage opportunities and also provides some early warnings for policy makers to manage volatility risks arising from carbon markets. In addition, this study contributes to the general development of stochastic process theory, and its applications in the following aspects are shown in Figure 1. We provide theoretical models and empirical conclusions to carbon price stochastic process of complex time series with nonstationary and nonlinear characteristics.

The remainder of this study is structured as follows. Section 2 gives a detailed introduction of the FIG-SVM 


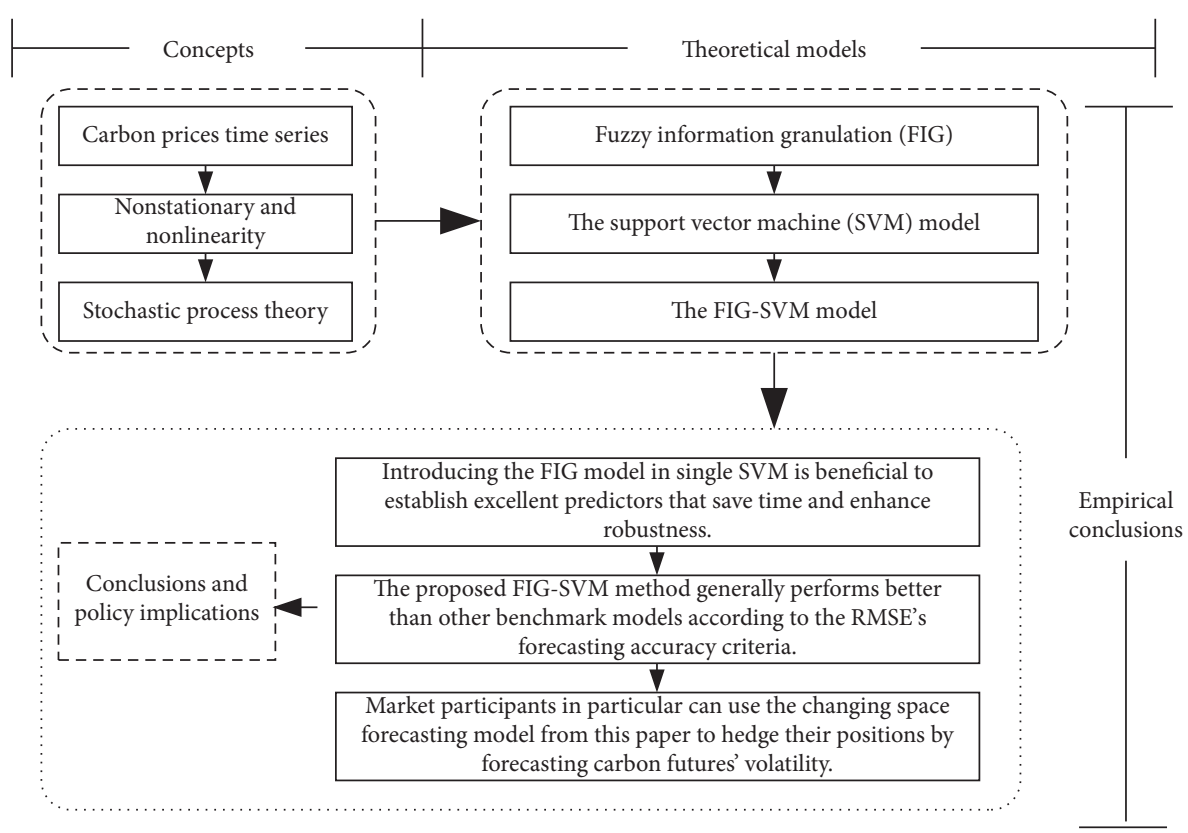

FIgURE 1: The contributions to stochastic process theory and its applications.

model. Section 3 illustrates some statistical characteristics of EUA data and essential testing results. Section 4 displays some valuable results from our proposed FIG-SVM model. Finally, the conclusion is set forth in Section 5.

\section{Methodology}

2.1. Fuzzy Information Granulation (FIG). Granular computing has become a general platform to construct, describe, and process information granules. It aims to discover an efficient system dealing with granulation in complex time series issues and has become a rapidly growing research field $[29,30]$. As carbon prices follow a stochastic process of complex time series with the inherent nonlinear and nonstationary characteristics $[3,31]$, it is necessary to granulate its information. The granulating process divides large amounts of complex information into several blocks according to their own characteristics, with each block defined as an information granule. Therefore, complex issues in a large-scale time series can be decomposed into simple ones by removing some irrelevant information.

Suppose that $X=\left\{x_{1}, x_{2}, \ldots x_{n}\right\}$ denotes a given time series of carbon prices and $p(1 \leq p \leq n)$ refers to time windows' number that determine the essential information retaining ability. The fuzzification process creates a convex fuzzy subset $G$ that can logically describe $X$. The formula is shown below:

$$
g \triangleq(x \text { is } G) \text { is } \lambda \text {, }
$$

where $\lambda$ is the probability of $x$ belonging to the subset $G$. Subsequently, if the changing space of future carbon prices is considered as an information granule, a model can be constructed to forecast this changing space for a future period. This model can better assist investors in making reasonable decisions compared to those based on points forecasting with numerical values. This paper adopts the triangular membership function proposed by Pedrycz and Vukovich [32]:

$$
T(x ; a, m, b)= \begin{cases}0, & x \leq a, \\ \frac{x-a}{m-a}, & a<x<m, \\ \frac{x-b}{m-b}, & m \leq x<b, \\ 0, & x \geq b,\end{cases}
$$

where $a, m$, and $b$ are the scalar parameters, corresponding to three variables Low, $R$, and $\mathrm{Up}$ for single window, which are obtained by granulation. The variable Low, $R$, and $\mathrm{Up}$, respectively, represent the minimum, average, and maximum value of the time series change in the window.

For a given time series $Y=\left\{Y_{i}, \ldots, Y_{n}\right\}$, a proper triangular granule $T(a, m, b)$ can be constructed by an optimization technique as follows:

$$
\max _{a, m, b} \sum_{Y_{i} \in Y} \frac{T\left(Y_{i} ; a, m, b\right)}{\text { measure }(\operatorname{Supp}(T(a, m, b)))},
$$

where measure $(\operatorname{Supp}(\mathrm{T}))$ is the measure of support of $T(a, m, b)$ while $T\left(Y_{i}\right)$ is the membership degree of $Y_{i}$ :

$$
T\left(Y_{i} ; a, m, b\right)= \begin{cases}\frac{x-a}{m-a}, & x<m, \\ \frac{x-b}{m-b}, & x \geq m .\end{cases}
$$

As for how to obtain an proper granule, we can solve the optimization equation (3) by minimizing the denominator 
measure (Supp (T)) and maximizing the numerator $T\left(Y_{i}\right)$ to acquire a high specificity of the granule that contain enough empirical information.

2.2. The Support Vector Machine (SVM) Model. As a machine-learning method based on statistical theory, SVM has been used to successfully address nonlinear classifications and regressions [19]. In this study, we focus on the SVM model in regression and forecasting for time series. Suppose that $T=\left\{\left(x_{1}, y_{1}\right), \ldots\left(x_{l}, y_{l}\right)\right\}$ is a training set of time series data, where $x_{i}$ and $y_{i}$ refer to input and output variables. They are defined by a regression model as follows:

$$
y=f\left(x_{i}\right)=\left(w \cdot x_{i}\right)+b,
$$

where $w$ is the weight vector and $b$ is the threshold. We can solve the optimization problem as follows to estimate $w$ and $b$ :

$$
\begin{array}{ll}
\min & \frac{1}{2}\|w\|^{2}+C \sum_{i=1}^{l}\left(\xi_{i}+\xi_{i}^{*}\right) \\
\text { s.t. } & \left(w \cdot x_{i}+b\right)-y_{i} \leq \varepsilon+\xi_{i} \\
& y_{i}-\left(w \cdot x_{i}+b\right) \leq \varepsilon+\xi_{i}^{*} \\
& \xi_{i}^{(*)} \geq 0, \quad i=1, \ldots, l,
\end{array}
$$

where $\|w\|^{2}$ is regularization term, $C$ is punishment coefficient, $\xi_{i}$ and $\xi_{i}^{*}$ are slack variables, and $\varepsilon$ is insensitive loss coefficient.

Due to the limited computing power of linear functions, nonlinear SVM becomes an extension for complicated timeseries forecasting issues. In order to get the nonlinear SVM, it is essential to perform a transformation as $\phi: R^{n} \longrightarrow H, x_{i} \longrightarrow \phi(x)$, making $K\left(x, x^{\prime}\right)=\phi(x) \cdot \phi(x)^{\prime}$, where $H$ denotes the high-dimensional feature space obtained by mapping the original space $x$ and $K\left(x, x^{\prime}\right)$ is a kernel function. In constructing a nonlinear SVM, the decision function takes the following form:

$$
y=f\left(x_{i}\right)=\sum_{i=1}^{l}\left(\alpha_{i}-\alpha_{i}^{*}\right) K\left(x_{i}, x\right)+b,
$$

where $\alpha$ and $\alpha^{*}$ are Lagrange multipliers and $b$ is the nonzero intercept.

2.3. The FIG-SVM Model. Ruan et al. [21] mentioned two challenges in using normal SVM models. First, the original data are often complex and characterised by noise and outliers in some real-world applications, which may disturb the normal SVM's performance in classifications or regressions. Second, a class imbalance problem may occur when training imbalanced data. Furthermore, most of the normal SVM classifiers with balanced class distribution assumptions or equal misclassification costs may produce models biased toward a majority class that exhibit weak performance towards a minority class. In summary, problems with noise and imbalances may affect the SVM model's accuracy and efficiency, a topic of much recent attention.

To deal with this issue, Lin and Wang [33] apply fuzzy theory to reduce impacts of noises and the results show that it performs well. Following their study of fuzzy SVM, we develop a novel FIG-SVM model for nonstationary and nonlinear time series prediction. This model can decompose large-scale complex time-series issues into some simpler problems to be handled independently. The original data are firstly extracted with partition sets in order to train and test. Next, the training data are granulated by the FIG method as Low, $R$, and Up. These granules are then integrated into SVM as input variables to select the optimal parameter pairs. After getting proper granules and selecting optimal SVM parameters, we can input the testing data in the FIG-SVM model to forecast the changing space of carbon prices time series.

\section{Data}

3.1. Data Description. We use three time-series datasets, comprised of European Union allowances' spot prices and future prices most heavily traded under the EU ETS, with daily frequencies. These prices are benchmarks of the most frequent EUA exchanges executed in the European Union's carbon markets. All original carbon pricing data are derived from European Energy Exchange's website (http://www.eex. com). Analysing carbon prices from different trading products of the spot and future markets allows us to compare different time series to investigate the proposed forecasting model's general applicability and robustness.

Substantial research has examined the modelling of carbon prices prior to the 2008 financial crisis to demonstrate that carbon prices peaked in mid-2008 [34-37], or just before the global financial crisis occurred. However, the carbon prices after this period exhibit an opposite trend, in which they sharply decline for various reasons. We investigate the price variations in the postcrisis era, which has demonstrated no such additional collapse, by choosing a carbon price time-series sample spanning 2009 to 2019. We select a dataset of EUA spot prices from 16 January 2009 to 22 March 2019, with 2,443 observations. Our EUA futures prices consist of two datasets: DEC18, which spans 26 March 2012 to 17 December 2018 and includes 1,705 observations; and DEC19, which spans 26 March 2012 to 26 March 26 2019, with 1,771 observations. The basic changing trends of these time series are illustrated in Figures 2-4.

Figure 2 plots the time variations of the EUA spot prices in our studied sample interval and displays the continuous EUA spot returns' volatility as computed by $r_{t}=\ln \left(p_{t}\right)-\ln \left(p_{t-1}\right)$. Clearly, the EUA spot prices' time series does not appear to be stationary; meanwhile, it exhibits violent fluctuations, decreasing by $53.65 \%$ from $€ 12.32$ on 16 January 2009 to $€ 5.71$ on 4 December 2012. This sharp decrease can be explained by some fundamentals, such as macroeconomic [38] or policy factors [39], among others. Furthermore, it increases by approximately 338\%, from $€ 5.71$ on 4 December 2012 to $€ 25.01$ on 22 January 2019. 

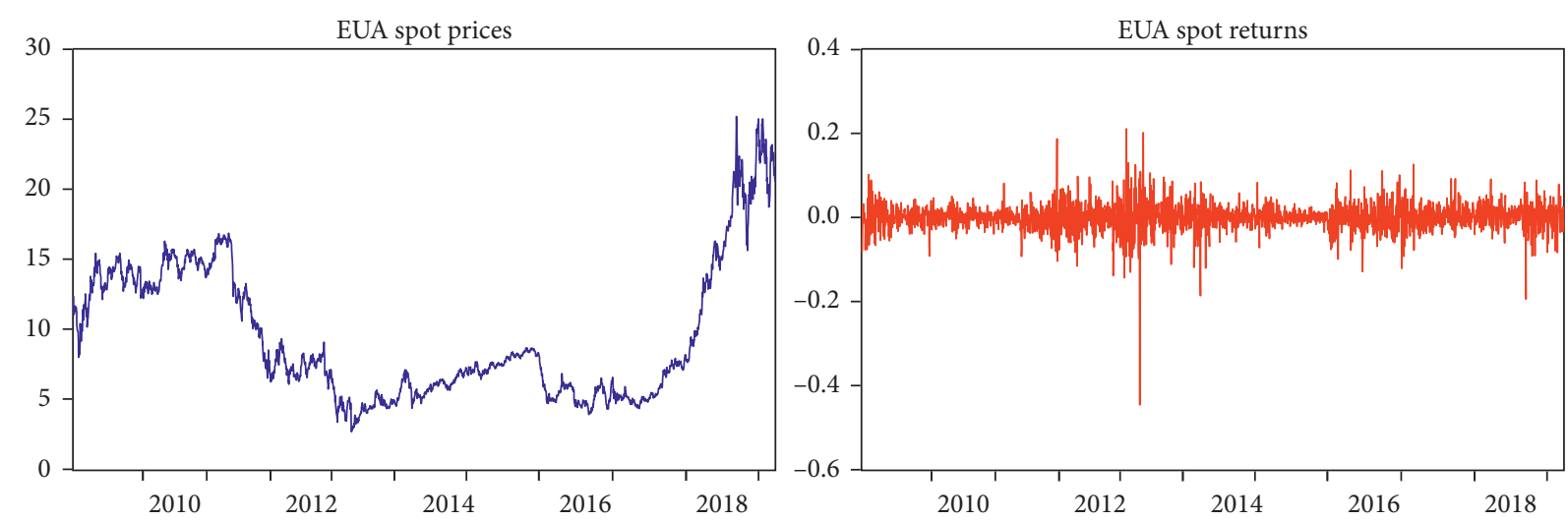

FIgURE 2: The time series of EUA spot prices and returns.

Regarding the EUA futures prices and returns from the DEC18 and DEC19 datasets, Figures 3 and 4 exhibit patterns of change similar to the EUA spot prices. This phenomenon occurs given the truth that these related markets have released some common information to investors. The correlation coefficient of spot prices and futures prices is significantly higher than 0.9 ; therefore, these series show a strong comovement while responding to the arrival of new common information.

Moreover, two noteworthy important features can be observed among the EUA spot and futures returns, plotted in Figures 2-4. On the one hand, the returns time series tendency towards volatility appears in bunches because information comes in clutters. This is because carbon returns' volatility is affected by previous values, and this is a persistent characteristic. Therefore, the time series future values can be easily forecast based on carbon returns' volatility. On the other hand, the most compelling feature is the leverage or asymmetric effect discovered early in financial markets according to the previous literature, a phenomenon that also exists in the carbon market [40, 41]. Clearly, carbon spot and future returns exhibit higher volatility when prices decrease than increase given an asymmetric response. This phenomenon appears in the form of negative impact arising from bad news, which may cause the increase in volatility to be greater than a positive impact from good news.

Table 1 presents the study's statistical indicators. The minimum and maximum values reveal that the changing range of daily EUA spot and futures prices is significantly large. Moreover, the huge intraday losses of spot and futures markets are obvious, with maximum losses of $44.66 \%$ in the EUA spot market, $36.38 \%$ in the DEC18 futures market, and $34.12 \%$ in the DEC19 futures market. Therefore, it is essential to establish a scientific changing spaceforecasting model for carbon prices. Furthermore, the coefficients of skewness and kurtosis exhibit a leptokurtic characteristic, and the returns on the EUA spot market and futures market are both negatively skewed. Revenues are significantly and negatively skewed which is attributed to the risk associated with high prices. From a distribution perspective, the carbon prices and returns are not normally distributed, but are characterised by "heavy-tailed" and "high-kurtosis" properties.
The result of Jarque-Bera test suggests that the "normally distributed" hypothesis can be rejected at any significance level, which is consistent with most prior studies [42-45]. This evidence suggests that forecasting the changing space of EUA spot and future prices is of great significance to risk managers and policy makers in carbon markets. As we have discovered some complicated statistics exist in carbon prices and returns series, the extra but essential tests are then performed to precisely determine these time series' nonstationary and nonlinear characteristics.

3.2. Nonstationary and Nonlinear Data Tests. This study adopts some classical time-series test methods to confirm the existence of nonstationary and nonlinear characteristics in carbon prices. First, it is necessary to test whether these time series are nonstationary. The augmented Dickey-Fuller (ADF) and Phillips-Perron tests are most commonly used in the existing literature based on the basic principle of unit root tests for nonstationary. Table 2 shows the ADF test result in Panel A and Phillips-Perron test result in Panel B, respectively. They demonstrate that EUA spot prices and futures prices are not stationary at the $1 \%, 5 \%$, and $10 \%$ significance levels.

However, these tests are not sufficiently convincing under abnormal circumstances. The traditional unit root test method can only identify the existence of unit roots in time series containing intercept or trend items and cannot identify the unit roots if breakpoints exist in the time series. If the stationary time series contains breakpoints, it is highly likely that a null hypothesis to accept nonstationarity will be obtained by the ADF or Phillips-Perron tests, distorting the test results; specifically, we should consider a case with structural breakpoints.

Prior literature indicates that the fluctuation of carbon prices has breakpoints, and the carbon prices' structure changes before and after these breakpoints [46, 47]. When these structural breakpoints exist in carbon prices, the power of conventional unit root tests as previously mentioned is unstable. Therefore, it is essential to use a breakpoint unit root test to examine the nonstationarity of this study's carbon price time series. Panel $\mathrm{C}$ in Table 2 lists the nonstationary breakpoint unit root test results, using a breakpoint ADF test for EUA spot and futures prices. 

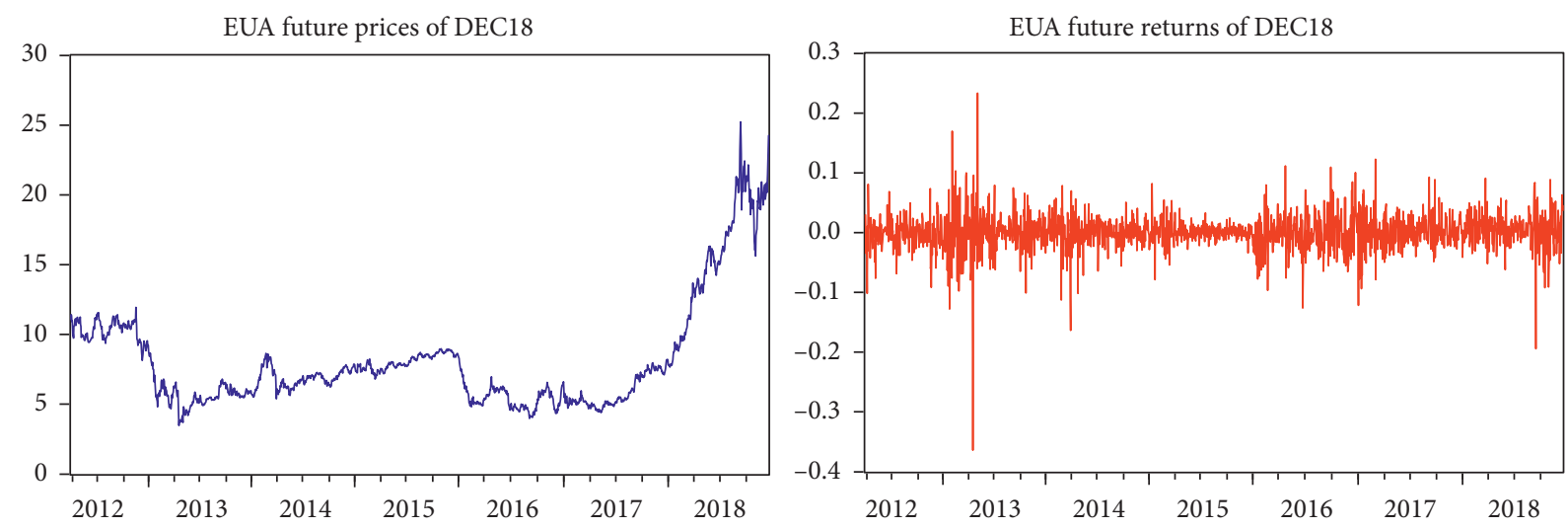

Figure 3: The time series of EUA future prices and returns for DEC18 contracts.
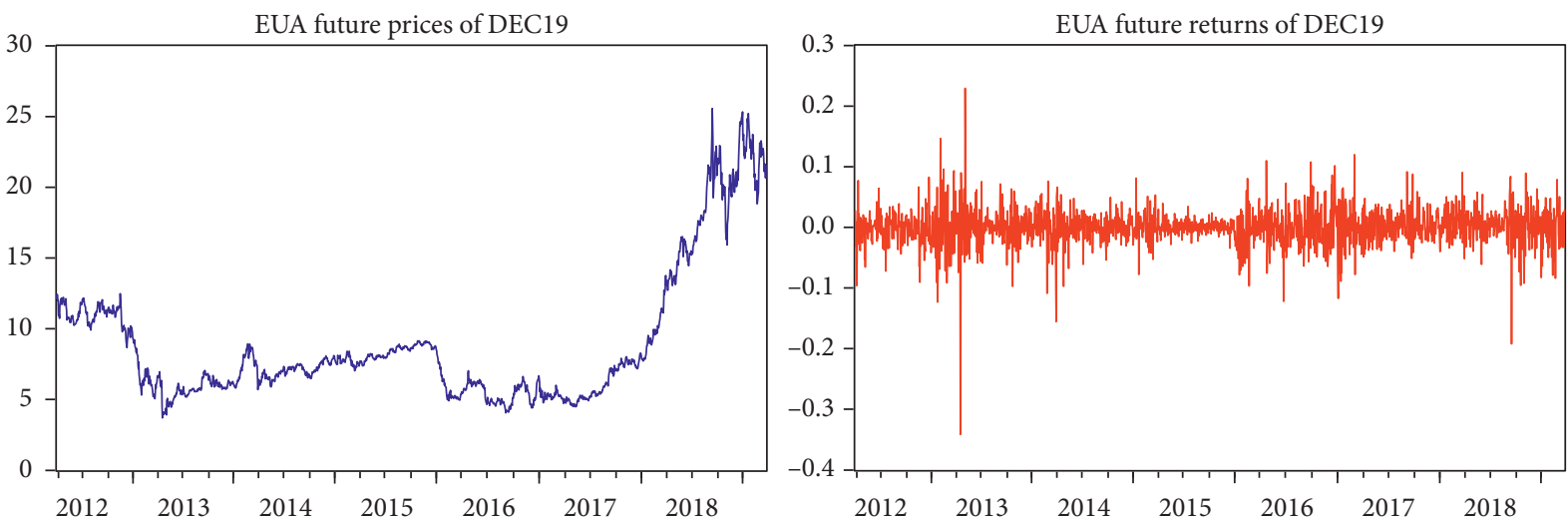

Figure 4: The time series of EUA future prices and returns for DEC19 contracts.

TABLE 1: Summary statistics of the EUA prices' and returns' time series.

\begin{tabular}{lcccccc}
\hline & \multicolumn{2}{c}{ EUA spot } & \multicolumn{2}{c}{ EUA futures-DEC18 } & \multicolumn{2}{c}{ EUA futures-DEC19 } \\
& Prices & Returns & Prices & Returns & Prices & Returns \\
\hline Mean & 9.5310 & 0.0002 & 8.1587 & 0.0005 & 8.9314 & 0.0003 \\
Median & 7.5950 & 0.0000 & 7.1150 & 0.0011 & 7.4400 & 0.0011 \\
Maximum & 25.1900 & 0.2106 & 25.2400 & 0.2334 & 25.5600 & 0.2292 \\
Minimum & 2.6800 & -0.4466 & 3.4800 & -0.3638 & 3.7100 & -0.3412 \\
Std. dev. & 4.8402 & 0.0329 & 3.8572 & 0.0319 & 4.7028 & 0.0311 \\
Skewness & 0.9405 & -0.9881 & 1.9159 & -0.9703 & 1.7496 & -0.8960 \\
Kurtosis & 3.0575 & 20.4610 & 6.4729 & 17.4954 & 5.3223 & 15.7228 \\
Jarque-Bera & 360.3567 & 31419.3800 & 1898.8660 & 15185.5700 & 1300.7370 & 12174.6200 \\
\hline
\end{tabular}

These results show that each of time series has a unit root; thus, we should accept the nonstationary hypothesis. This study's breakpoint ADF test adopts an innovational outlier (IO) model by assuming that the breakpoint process is gradual and an exogenous shock follows the same dynamic process. Two structural breakpoints were found on 5 September 2017 and 15 January 2018, according to a selection method that maximised the $t$-statistic of the intercept term's breakpoint.

Moreover, it is also important to test the EUA spot and futures prices' nonlinear characteristics. Our nonlinearity test of the time series adopts the Brock--Decher-Scheikman test method used by Zhu et al. [17]; with two to six embedding dimensions. Table 3 presents the nonlinearity test results and demonstrates that our studied sample data are not linear. Our findings are consistent with those of other studies determining that the carbon prices time series follow a nonlinear process $[8,9,10,17]$.

This nonlinearity test leads us to conclude that carbon prices cannot be linearly predicted using traditional models, as this will lead to distortions once the linear model is established for nonlinear data. To deal with this issue, we apply an SVM model developed through statistical learning theory in a nonlinear regression estimation. Furthermore, a structural risk-minimisation principle is adopted for the function estimation by minimising the upper boundary of some generalisation errors [48]. This method can also create favourable effects in resisting the overfitting problem, which 
Table 2: The ADF test, Phillips-Perron test, and Breakpoint ADF test results for nonstationary time series.

\begin{tabular}{|c|c|c|c|}
\hline & EUA spot & EUA futures-DEC18 & EUA futures-DEC19 \\
\hline \multicolumn{4}{|c|}{ Panel A: ADF test } \\
\hline t-statistic & -0.8185 & 0.8239 & -0.2305 \\
\hline Prob. & 0.8132 & 0.9945 & 0.9321 \\
\hline \multicolumn{4}{|l|}{ Critical values: } \\
\hline $1 \%$ & -3.4328 & -3.4340 & -3.4338 \\
\hline $5 \%$ & -2.8625 & -2.8630 & -2.8630 \\
\hline $10 \%$ & -2.5673 & -2.5676 & -2.5676 \\
\hline \multicolumn{4}{|c|}{ Panel B: Phillips-Perron test } \\
\hline Adj. t-Statistic & -0.6770 & 1.6015 & 0.1372 \\
\hline Prob. & 0.8505 & 0.9995 & 0.9684 \\
\hline \multicolumn{4}{|l|}{ Critical values: } \\
\hline $1 \%$ & -3.4328 & -3.4340 & -3.4338 \\
\hline $5 \%$ & -2.8625 & -2.8630 & -2.8630 \\
\hline $10 \%$ & -2.5673 & -2.5676 & -2.5676 \\
\hline \multicolumn{4}{|c|}{ Panel C: Breakpoint ADF test } \\
\hline t-Statistic & -3.1497 & -4.3648 & -3.8930 \\
\hline Prob. & 0.6151 & 0.2706 & 0.5113 \\
\hline \multicolumn{4}{|l|}{ Critical values: } \\
\hline $1 \%$ & -5.1497 & -5.7114 & -5.7114 \\
\hline $5 \%$ & -4.6106 & -5.1550 & -5.1550 \\
\hline $10 \%$ & -4.3073 & -4.8610 & -4.8610 \\
\hline
\end{tabular}

TABLE 3: The Brock-Decher-Scheikman (BDS) test results for nonlinear time series.

\begin{tabular}{lccccr}
\hline \multirow{2}{*}{ Dimension } & \multicolumn{2}{c}{ EUA spot } & \multicolumn{2}{c}{ EUA futures-DEC18 } & \multicolumn{2}{c}{ EUA futures-DEC19 } \\
& BDS statistic & Prob. & BDS statistic & Prob. & BDS statistic \\
\hline 2 & 0.1974 & 0.0000 & 0.1994 & 0.0000 & 0.2006 \\
3 & 0.3357 & 0.0000 & 0.3391 & 0.0000 & 0.3416 \\
4 & 0.4320 & 0.0000 & 0.4366 & 0.0000 & 0.4403 \\
5 & 0.4987 & 0.0000 & 0.5046 & 0.0000 & 0.0000 \\
6 & 0.5445 & 0.0000 & 0.5519 & 0.0000 & 0.0000 \\
\hline
\end{tabular}

may create imprecise and unsatisfactory forecasting. So far, the application of SVM to nonlinear regression estimation problems has become one of the most successful technical cases.

\section{Empirical Results}

4.1. Extracting Sample Data. We divide the entire dataset into two subsets to test the proposed forecasting model's validity in light of Alizadeh et al. 's [49] study. The first twothirds of the total sample data were selected as a dataset to train our model and obtain the optimal parameter estimation. The last one-third of all sample were utilized as a testing set to forecast the future carbon prices based on the established model. Table 4 reports the details of the partitioned data set.

4.2. Fuzzy Information Granulation (FIG). We use the fuzzy granulation model as proposed in Section 2.1 to granulate the original data, which divides a complicated problem into some simpler ones to be handled by our proposed granular computing technique. This basic process consists of four steps, as illustrated in Figure 5.

These four steps will granulate the original time series of carbon prices into the following granules: Low (minimum),
$R$ (average), and Up (maximum). First, Low is generated by granulating the relatively small values of each window, which can denote the minimum values of original data changes. Second, $R$ is generated from the average values of each granulated window, indicating the major dominant of original data changes. Third, Up is produced from the larger values of each granulated window, denoting the maximum values of the original data changes.

The three variables-Low, $R$, and Up-are obtained after the fuzzy granulation process for each window, as Figure 6 illustrates. These variables, respectively, correspond to the three parameters of $a, m$, and $b$ in the triangular fuzzy granulation presented in equation (2). The normal SVM input size decreases for the carbon prices' time series after granulation, which may improve forecasting efficiency. Therefore, the FIG theory is successfully incorporated into a normal SVM model to develop time saving, robust predictors. The proposed hybrid FIG-SVM model can produce results with a good fit in a shorter time, which is advantageous for large scale, nonstationary, and nonlinear time series.

4.3. The Parameter Optimization. The model's generalisability can only be tested after obtaining a trained model with appropriate parameters. It is necessary to strive for selecting 
TABLE 4: The division results of training set and testing set of the total sample.

\begin{tabular}{lccc}
\hline Carbon prices & & Size & Date \\
\hline \multirow{3}{*}{ EUA spot } & Sample set & 2443 & January 16, 2009-March 22, 2019 \\
& Training set & 1629 & January 16, 2009-January 13, 2016 \\
& Testing set & 814 & January 14, 2016-March 22, 2019 \\
\hline \multirow{3}{*}{ EUA futures-DEC18 } & Sample set & 1705 & March 26, 2012-December 17, 2018 \\
& Training set & 1137 & March 26, 2012-September 27, 2016 \\
& Testing set & 568 & September 28, 2016-December 17, 2018 \\
\hline \multirow{3}{*}{ EUA futures-DEC19 } & Sample set & 1771 & March 26, 2012-March 26, 2019 \\
& Training set & 1181 & March 26, 2012-November 28, 2016 \\
& Testing set & 590 & November 29, 2016-March 26, 2019 \\
\hline
\end{tabular}

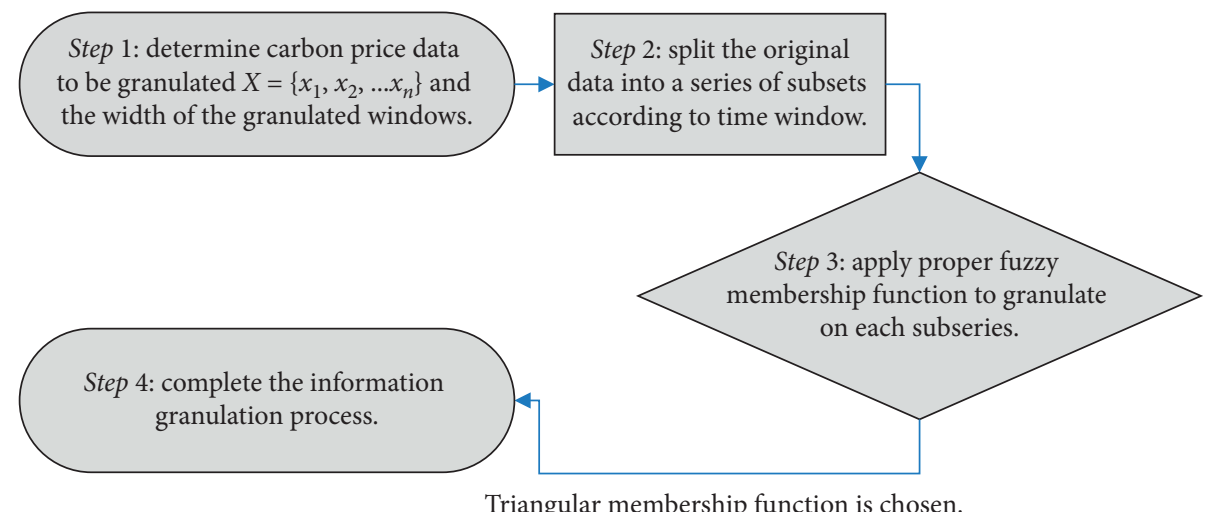

FIgURE 5: The basic steps of FIG on the original time series.
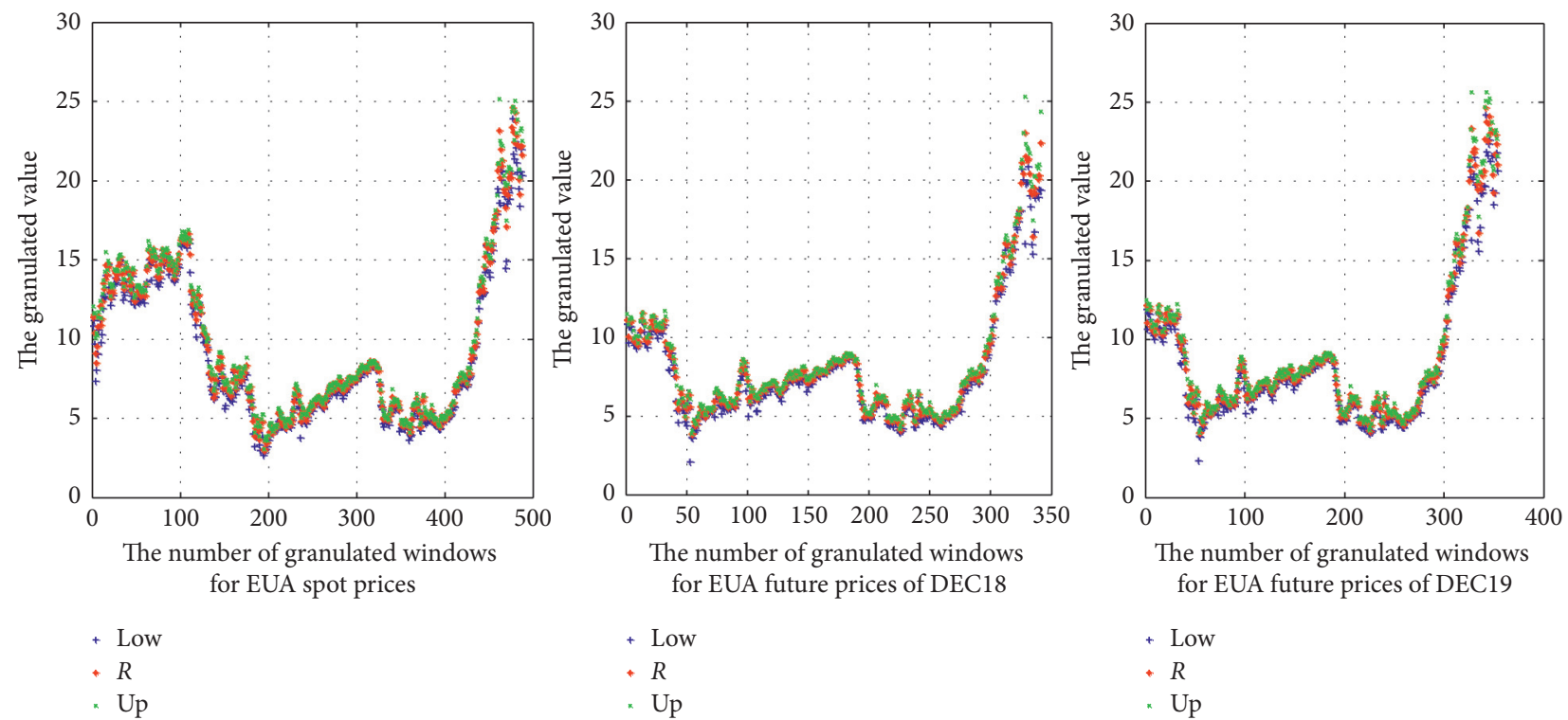

Figure 6: The granulation results for the original EUA spot and futures prices time series.

optimal SVM parameters because it greatly affects the quality of support machine regression for prediction. This selection process involves two crucial parameter pairs: the penalty parameter $c$ and kernel parameter $g$ (abbreviated for $c-g$ ). While no internationally recognised or unified method exists in the optimal parameter selection process for SVM, a more common method involves calculating $c-g$ parameter pairs within a given scope. For example, a training set for a selected $c-g$ parameter pairs is taken as an original dataset, and a crossvalidating $\mathrm{K}$-fold crossvalidation method (K-CV) is applied to obtain the training accuracy of the dataset under the selected $c-g$ parameter pairs. Finally, the selected $c-g$ parameter pairs achieving maximum precision in the training set is taken as the best parameters for SVM model. 
However, an unexpected issue may occasionally occur, in which multiple pairs of $c-g$ may correspond to the maximum precision. This study's method to address this issue involves selecting the parameter pairs with the smallest $c$ to attain the maximum precision. If multiple pairs of $g$ correspond to the smallest $c$, the first $c-g$ parameter we searched is selected as the best one. This is due to the following facts. The penalty parameter that is too high will lead to overlearning, or specifically, the training and testing sets exhibit very high and very low accuracies, respectively, or generalisability decreases. Therefore, the smaller penalty parameter is considered as a more reasonable selection in all the $c-g$ parameter pairs that can achieve the highest verification accuracy. Table 5 displays the crossvalidation results for optimising SVM parameters.

The Low parameter in the EUA spot prices' time series can be taken as an example to explain the SVM for regression prediction process in detail. The optimal parameter combination under the crossvalidation condition is obtained as the relaxation parameter $c=1$ and the kernel parameter $g=0.0055$. Figure 7 illustrates the optimisation parameterselection results for the Low parameters. The left and right panels display the contour map and the 3D view, respectively, which verify that the SVMs' parameters significantly impact their regression results. The other optimal parameter-searching processes for $R$ and Up in the EUA spot and futures prices' time series yield similar results, which are omitted here for brevity.

4.4. Predict the Low (Minimum), $R$ (Average), and up (Maximum). After obtaining the proper granules for Low (minimum), $R$ (average), and Up (maximum) and selecting the optimal $c-g$ parameter pairs for SVM, we input these into the SVM model to predict the possible ranges of change for the next window. Next, we take the EUA spot prices here as an example to demonstrate our proposed FIG-SVM results. Figure 8 illustrates the fitting results of the Low, $R$, and Up fuzzy particles for the EUA spot prices. The predict Low, predict $R$, and predict $U p$ time series depict the predicted values of the Low, $R$, and Up fuzzy particles, which are close to the original Low, original $R$ and original $U p$ time series exhibiting the actual values of the Low, $R$, and Up fuzzy particles, respectively.

The results indicate that the proposed FIG-SVM forecasting model performs well in the case involving EUA spot prices. We further validate this method's effectiveness by conducting more experiments on the other two time series. With the same settings and the same granulation window, we obtain the results from our proposed FIG-SVM model for the Low, $R$, and Up granules in the cases of DEC18 and DEC19 futures prices. These experimental results all exhibit predicted values that approximate real values. This indicates that the normal SVM model combined with a developed FIG method can produce well-fit performances for predicting carbon prices time series with complex characteristics.
TABLE 5: The SVM parameters' optimisation results.

\begin{tabular}{lccc}
\hline Carbon prices & & Best $c$ & Best $g$ \\
\hline \multirow{3}{*}{ EUA spot } & Low & 1 & 0.0055 \\
& $R$ & 2.8284 & 0.0055 \\
& $\mathrm{Up}$ & 64 & 0.0442 \\
\hline \multirow{3}{*}{ EUA futures-DEC18 } & Low & 2 & 0.0625 \\
& $R$ & 1 & 0.0313 \\
& $\mathrm{Up}$ & 64 & 0.0625 \\
\hline \multirow{2}{*}{ EUA futures_DEC19 } & Low & 1 & 0.0625 \\
& $R$ & 4 & 0.0884 \\
& $\mathrm{Up}$ & 181 & 0.0039 \\
\hline
\end{tabular}

4.5. Forecasting Performance Measures. We use ARIMA, ARFIMA, and Markov-switching method as benchmarks to compare the proposed FIG-SVM forecasting model's prediction capacity against other widely used forecasting approaches. As for evaluation criterion, we choose the root mean square error (RMSE) given by

$$
\mathrm{RMSE}=\sqrt{\frac{1}{N} \sum_{t=1}^{n}\left(\hat{y}_{t}-y_{t}\right)^{2}} .
$$

The formula represents the extent to which the predicted value of our proposed model deviates from actual data. The predicted value is going to be more consistent with the actual value, as the RMSE gets smaller.

Table 6 reveals that the proposed FIG-SVM forecasting model decreases prediction errors by $40.06 \%, 33.88 \%$, and $25.49 \%$ compared to the traditional ARIMA model to calculate the EUA spot, DEC18, and DEC19 futures time series, respectively. Moreover, our model performs better than the other forecasting models as ARFIMA and Markov switching. From this perspective, the proposed FIG-SVM forecasting model can produce a well-fit performance, which verifies the effectiveness of the FIG-SVM model to forecast complicated time series with the characteristics of nonstationarity and nonlinearity.

4.6. Carbon Prices Changing Space Forecasting. After obtaining the FIG-SVM training predictors, the testing data are used to forecast the changing space of carbon prices' time series and evaluate the forecast performance. As Table 7 indicates, SVM can be used to make regression predictions for the Low, $R$, and Up fuzzy granulations to obtain the parameters of Low, $R$, and Up within five trading days, respectively.

We then verified the FIG-SVM model predictions' effectiveness by testing whether the carbon prices in the next five days changed within the range of the previous prediction. We compared this with the previous five trading days to investigate the overall trends in carbon pricing changes. The results reveal that the predicted range of carbon prices' changes in the next five days is accurate. Moreover, the EUA spot price indicates a downward trend in the following five days compared with the previous five days. However, opposite results occur in cases of EUA futures price changes, while the DEC18 and DEC19 carbon prices 


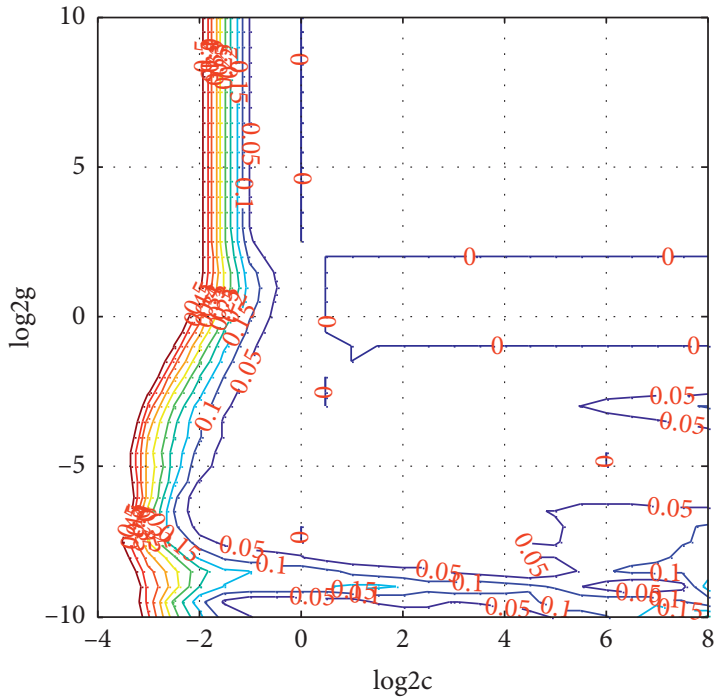

SVR parameter selection results (contour diagram)

(a)

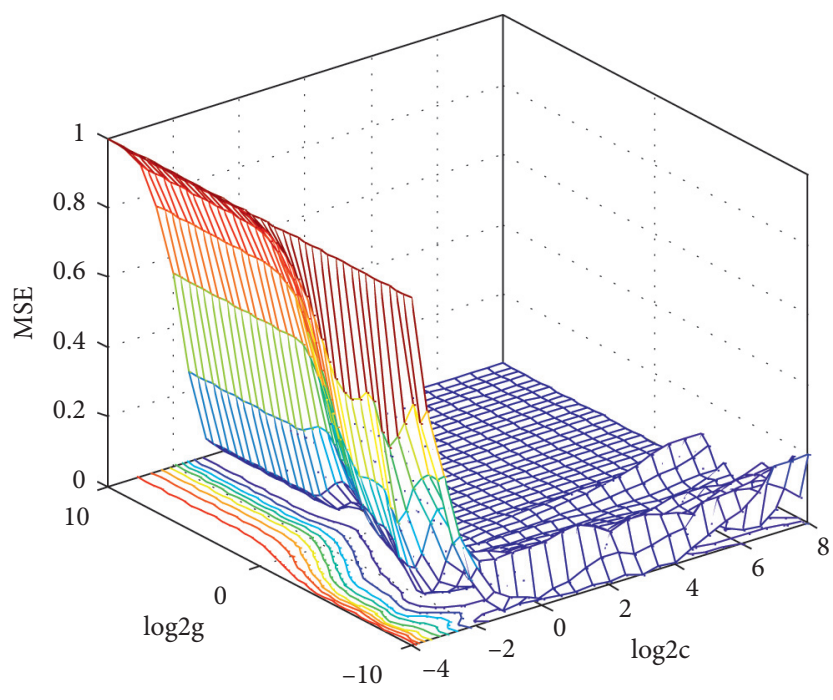

SVR parameter selection results (3D view)

(b)

Figure 7: The parameter selection results for Low (minimum) value of the EUA spot prices. (a) SVR parameter selection results (contour diagram). (b) SVR parameter selection results (3D view).
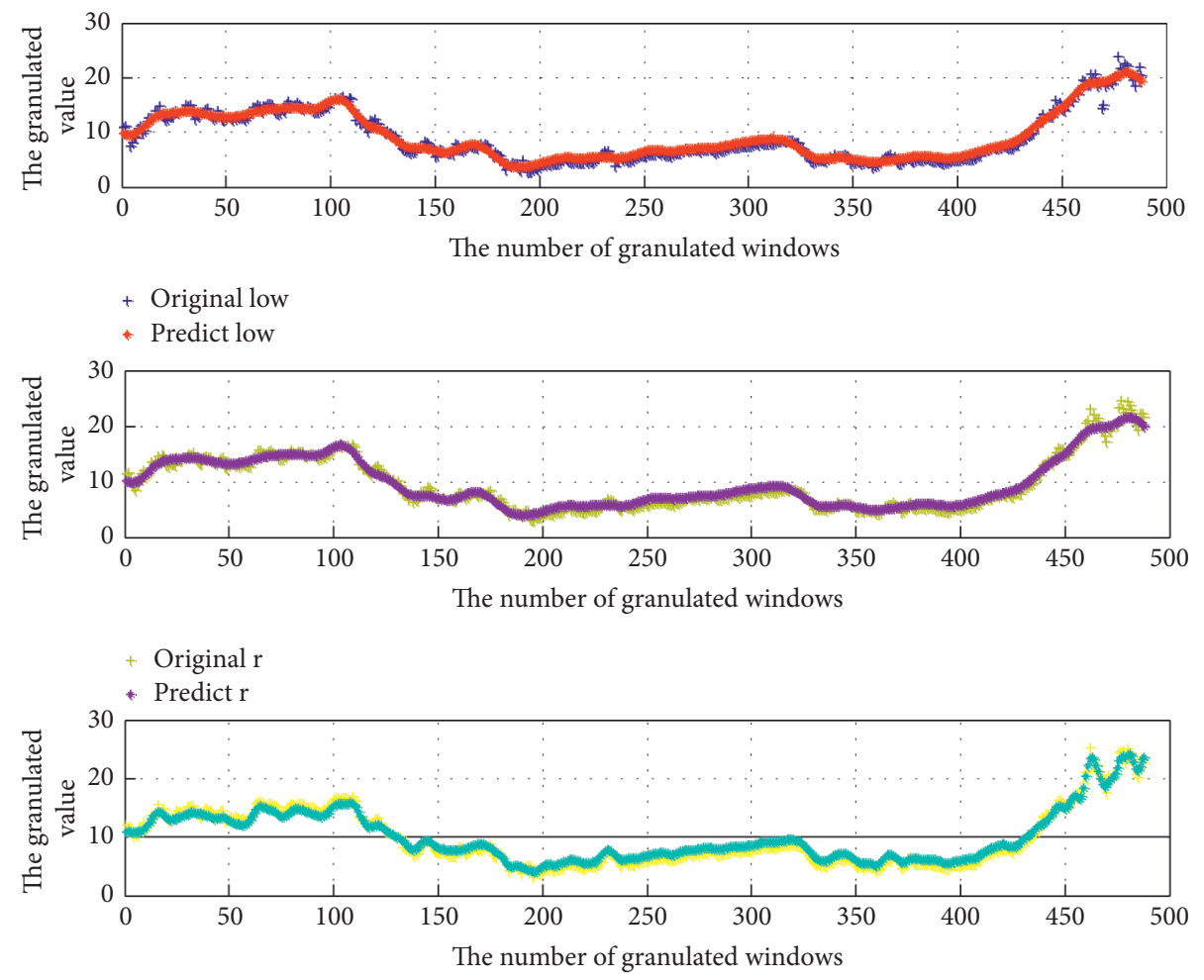

Original up

* Predict up

Figure 8: The prediction results for the Low, $R$, and Up of the EUA spot prices. 
TABLE 6: The comparison results of the proposed FIG-SVM model with traditional forecasting models.

\begin{tabular}{lccc}
\hline Forecasting models & EUA spot & EUA futures-DEC18 & EUA futures-DEC19 \\
\hline Panel A: Root Mean Square Error (RMSE) & & & \\
ARIMA & 0.031879 & 0.031678 & 0.030991 \\
ARFIMA & 0.031872 & 0.031690 & 0.030940 \\
Markov-switching & 0.031856 & 0.031577 & 0.030878 \\
FIG-SVM & 0.019108 & 0.020945 & 0.023091 \\
\hline Panel B: Error Reduction (\%) & & & 33.88 \\
FIG-SVM/ARIMA & 40.06 & 33.91 & 25.49 \\
FIG-SVM/ARFIMA & 40.05 & 33.67 & 25.37 \\
FIG-SVM/Markov-switching & 40.02 & & 25.22 \\
\hline
\end{tabular}

TABle 7: The changing space forecasting results for EUA spot and future prices.

\begin{tabular}{|c|c|c|c|c|c|c|c|}
\hline \multirow[t]{2}{*}{ Carbon prices } & \multicolumn{6}{|c|}{ The original data } & \multirow{5}{*}{$\begin{array}{c}\text { Changing space } \\
\text { Actual changing space } \\
{[\text { Low, } R, \mathrm{Up}]=[20.57,21,21.71]} \\
\text { Predicted changing space } \\
{[\text { Low } R, \mathrm{Up}]=[18.79,19.89,22.60]}\end{array}$} \\
\hline & Date & 2019/3/11 & $2019 / 3 / 12$ & $2019 / 3 / 13$ & $2019 / 3 / 14$ & $2019 / 3 / 15$ & \\
\hline \multirow{3}{*}{ EUA spot } & Price & 22.18 & 22.22 & 22.16 & 22.61 & 22.35 & \\
\hline & Date & $2019 / 3 / 18$ & 2019/3/19 & $2019 / 3 / 20$ & $2019 / 3 / 21$ & $2019 / 3 / 22$ & \\
\hline & Price & 21.71 & 21 & 21.52 & 20.82 & & \\
\hline \multirow{4}{*}{ EUA futures-DEC18 } & Date & $2018 / 12 / 3$ & $2018 / 12 / 4$ & $2018 / 12 / 5$ & $2018 / 12 / 6$ & $2018 / 12 / 7$ & Actual changing space \\
\hline & Price & 20.64 & 20.73 & 19.68 & 19.99 & 20.3 & {$[$ Low $, R, \mathrm{Up}]=[20.16,21.47,23.37]$} \\
\hline & Date & $2018 / 12 / 10$ & 2018/12/11 & 2018/12/12 & $2018 / 12 / 13$ & $2018 / 12 / 14$ & Predicted changing space \\
\hline & Price & 20.86 & 20.16 & 21.47 & 22.32 & 23.37 & {$[\mathrm{Low}, R, \mathrm{Up}]=[16.66,19.38,23.88]$} \\
\hline \multirow{4}{*}{ EUA futures-DEC19 } & Date & $2019 / 2 / 18$ & $2019 / 2 / 19$ & $2019 / 2 / 20$ & $2019 / 2 / 21$ & $2019 / 2 / 22$ & Actual changing space \\
\hline & Price & 20.02 & 20.2 & 20.47 & 18.82 & 18.93 & {$[$ Low $, R, \mathrm{Up}]=[19.25,21.28,22.28]$} \\
\hline & Date & $2019 / 2 / 25$ & $2019 / 2 / 26$ & $2019 / 2 / 27$ & $2019 / 2 / 28$ & $2019 / 3 / 1$ & Predicted changing space \\
\hline & Price & 19.25 & 19.67 & 21.28 & 21.69 & 22.28 & {$[\mathrm{Low}, R, \mathrm{Up}]=[17.05,17.86,23.66]$} \\
\hline
\end{tabular}

exhibit upward trends in the following five days compared with the previous five days.

\section{Conclusions}

In this paper, the data of EUA spot prices and futures prices for DEC18 and DEC19 from 2009 to 2019 are examined for changing space forecasting of carbon prices. Since the launch of the European Union ETS, its rules have changed significantly from the first stage of 2005-2007 to the third stage of 2013-2020. The first stage is a pilot period, and the second stage is related to stricter emission caps because some industrialized countries have already committed to binding emission targets. The third stage has undergone some specific changes. Under the influence of different rules, the carbon prices in different stages of the EU emission trading system may change significantly. From a new perspective, this paper proposes a model that can predict the fluctuation space of carbon prices, which is of great significance to investors and policy makers involved in carbon market trading. It helps them to understand the changing laws of carbon prices and establish an effective carbon price stabilization mechanism. Furthermore, it can avoid carbon market risks and therefore enhance the activity of carbon trading participants. Some conclusions are drawn as follows.

First, we provide a changing space-forecasting model for carbon prices, which existing studies of carbon price forecasting have neglected. Prior literature has focused on a point prediction method for carbon prices, although relatively few works have forecast the changing space for carbon prices' time series. In fact, changing space forecasting is more important in practical investment applications, as investors can decide to buy a call or put options according to the predicted changing space. Moreover, investors can estimate the maximum or minimum loss or return in advance according to the Low, $R$, and Up fuzzy granulations. This article provides several important implications for investors and risk managers involved in trading carbon financial products. Market participants in particular can use the changing space forecasting model from this article to hedge their positions by forecasting carbon futures' volatility.

Second, we develop an integrated FIG-SVM approach for changing space forecasting, which builds a foundation to solve decision-making problems. Introducing the FIG model in single SVM is beneficial to establish excellent predictors that save time and enhance robustness. The granulation method produces Low (minimum), $R$ (average), and Up (maximum) granules from carbon prices' time series. It can decompose large-scale complex time series issues into some simpler problems to be handled independently. Thus, the FIG method improves the prediction efficiency by reducing the complexity of the data and retaining the inherent essential information.

Third, we compare the forecasting performances between the proposed FIG-SVM model and traditional ARIMA, ARFIMA, and Markov-switching models in the context of carbon prices changing space forecasting. The empirical experimental results reveal that the proposed FIGSVM method generally performs better than other benchmark models according to the RMSE's forecasting accuracy criteria. We prove the FIG-SVM model's feasibility and effectiveness in forecasting the changing space of carbon 
prices with some complicated characteristics of nonstationary and nonlinearity.

Although this paper provides the previously mentioned contributions, other issues still remain for further study. The complex changes and fluctuations in carbon prices is a nonlinear, dynamic system problem characterised by randomness, significant noise, and strong nonlinearity, among other chaotic characteristics. Such a system tends to exhibit unstable increases and decreases, resulting in a high concentration of risk. Therefore, the chaotic nonlinear dynamic system model is one alternative to analyse the characteristics of market price behaviour. This may lead to a new direction in studying the essential characteristics of carbon prices' fluctuation. An additional important issue pertains to China's well-known carbon-trading system, which has rapidly developed among emerging markets in recent years. However, the relevant literature is insufficient and must be further strengthened in the future.

\section{Data Availability}

The data used to support the findings of this study are available from the corresponding author upon request.

\section{Conflicts of Interest}

The authors declare that they have no conflicts of interest.

\section{Acknowledgments}

This work was supported by the National Natural Science Foundation of China (nos. 71704098, 71974154, and 71701115) and Natural Science Foundation of Shandong Province (nos. ZR2016GQ03, ZR2017QG003, and ZR2017MF058).

\section{References}

[1] Z. H. Feng, Y. M. Wei, and K. Wang, "Estimating risk for the carbon market via extreme value theory: an empirical analysis of the EU ETS," Applied Energy, vol. 99, pp. 97-108, 2012.

[2] Y. Zhang and Y. Wei, "An overview of current research on EU ETS: evidence from its operating mechanism and economic effect," Applied Energy, vol. 87, pp. 1804-1814, 2010.

[3] W. Sun and C. Zhang, "Analysis and forecasting of the carbon price using multi-resolution singular value decomposition and extreme learning machine optimized by adaptive whale optimization algorithm," Applied Energy, vol. 231, pp. 13541371, 2018.

[4] E. Benz and S. Trück, "Modeling the price dynamics of $\mathrm{CO}_{2}$ emission allowances," Energy Economics, vol. 31, no. 1, pp. 4-15, 2009.

[5] A. K. Dhamija, S. S. Yadav, and P. K. Jain, "Forecasting volatility of carbon under EU ETS: a multi-phase study," Environmental Economics and Policy Studies, vol. 19, pp. 299-335, 2017.

[6] C. García-Martos, J. Rodríguez, and M. J. Sánchez, "Modelling and forecasting fossil fuels, $\mathrm{CO} 2$ and electricity prices and their volatilities," Applied Energy, vol. 101, pp. 363-375, 2013.

[7] M. E., Sanin, F. Violante, and M. Mansanet-Bataller, "Understanding volatility dynamics in the EU ETS market," Energy Policy, vol. 82, pp. 321-331, 2015.
[8] M. E. H. Arouri, F. Jawadi, and D. K. Nguyen, "Nonlinearities in carbon spot-futures price relationships during phase II of the EU ETS," Economic Modelling, vol. 29, no. 3, pp. 884-892, 2012.

[9] J. Chevallier, "Nonparametric modeling of carbon prices," Energy Economics, vol. 33, no. 6, pp. 1267-1282, 2011.

[10] B. J. Lutz, U. Pigorsch, and W. Rotfuß, "Nonlinearity in capand-trade systems: the EUA price and its fundamentals," Energy Economics, vol. 40, pp. 222-232, 2013.

[11] G. S. Atsalakis, "Using computational intelligence to forecast carbon prices," Applied Soft Computing, vol. 43, pp. 107-116, 2016.

[12] X. Fan, S. Li, and L. Tian, "Chaotic characteristic identification for carbon price and an multi-layer perceptron network prediction model," Expert Systems with Applications, vol. 42, pp. 3945-3952, 2015.

[13] M. Han, L. Ding, X. Zhao, and W. Kang, "Forecasting carbon prices in the Shenzhen market, China: the role of mixedfrequency factors," Energy, vol. 171, pp. 69-76, 2019.

[14] J. Zhang, D. Li, Y. Hao, and Z. Tan, "A hybrid model using signal processing technology, econometric models and neural network for carbon spot price forecasting," Journal of Cleaner Production, vol. 204, pp. 958-964, 2018.

[15] B. Zhu, D. Han, P. Wang, Z. Wu, T. Zhang, and Y. Wei, "Forecasting carbon price using empirical mode decomposition and evolutionary least squares support vector regression," Applied Energy, vol. 191, pp. 521-530, 2017.

[16] B. Zhu and Y. Wei, "Carbon price forecasting with a novel hybrid ARIMA and least squares support vector machines methodology," Omega, vol. 41, pp. 517-524, 2013.

[17] B. Zhu, S. Ye, P. Wang, K. He, T. Zhang, and Y. Wei, "A novel multiscale nonlinear ensemble leaning paradigm for carbon price forecasting," Energy Economics, vol. 70, pp. 143-157, 2018.

[18] J. Zhu, P. Wu, H. Chen, J. Liu, and L. Zhou, "Carbon price forecasting with variational mode decomposition and optimal combined model," Physica AStatistical Mechanics and its Applications, vol. 519, pp. 140-215, 2019.

[19] C. Cortes and V. N. Vapnik, "Support vector networks," Machine Learning, vol. 20, no. 3, pp. 273-297, 1995.

[20] Y. Song, T. Liu, D. Liang, Y. Li, and X. Song, "A fuzzy stochastic model for carbon price prediction under the effect of demand-related policy in China's carbon market," Ecological Economics, vol. 157, pp. 253-265, 2019.

[21] J. Ruan, X. Wang, and Y. Shi, "Developing fast predictors for large-scale time series using fuzzy granular support vector machines," Applied Soft Computing, vol. 13, no. 9, pp. 3981-4000, 2013.

[22] C. Tian and Y. Hao, "Point and interval forecasting for carbon price based on an improved analysis-forecast system," Applied Mathematical Modelling, vol. 79, pp. 126-144, 2020.

[23] L. A. Zadeh, "Fuzzy sets and information granularity," Advances in Fuzzy Set Theory and Application, vol. 1, pp. 3-18, 1979.

[24] M. Y. Chen and B. T. Chen, "A hybrid fuzzy time series model based on granular computing for stock price forecasting," Information Sciences, vol. 294, pp. 227-241, 2015.

[25] Y. He, Y. Yan, X. Wang, and C. Wang, "Uncertainty forecasting for streamflow based on support vector regression method with fuzzy information granulation," Energy Procedia, vol. 158, pp. 6189-6194, 2019.

[26] O. Hryniewicz and K. Kaczmarek, "Bayesian analysis of time series using granular computing approach," Applied Soft Computing Journal, vol. 47, pp. 644-652, 2016. 
[27] W. Lu, X. Chen, W. Pedrycz, X. Liu, and J. Yang, "Using interval information granules to improve forecasting in fuzzy time series," International Journal of Approximate Reasoning, vol. 57, pp. 1-18, 2015.

[28] L. Wang, X. Liu, and W. Pedrycz, "Effective intervals determined by information granules to improve forecasting in fuzzy time series," Expert Systems with Applications, vol. 40, no. 14, pp. 5673-5679, 2013.

[29] B. Huang, Y. L. Zhuang, and H. X. Li, "Information granulation and uncertainty measures in interval-valued intuitionistic fuzzy information systems," European Journal of Operational Research, vol. 231, no. 1, pp. 162-170, 2013.

[30] W. Pedrycz, "Allocation of information granularity in optimization and decision-making models: towards building the foundations of Granular Computing," European Journal of Operational Research, vol. 232, no. 1, pp. 137-145, 2014.

[31] Y. Huang and Z. He, "Carbon price forecasting with optimization prediction method based on unstructured combination," Science of the Total Environment, vol. 725, no. 10, p. 138350, 2020.

[32] W. Pedrycz and G. Vukovich, "Feature analysis through information granulation and fuzzy sets," Pattern Recognition, vol. 35, no. 4, pp. 825-834, 2002.

[33] C. F. Lin and S. D. Wang, "Training algorithms for fuzzy support vector machines with noisy data," Pattern Recognition Letters, vol. 25, no. 14, pp. 1647-1656, 2004.

[34] J. Chevallier, "Carbon futures and macroeconomic risk factors: a view from the EU ETS," Energy Economics, vol. 31, no. 4, pp. 614-625, 2009.

[35] G. Daskalakis, D. Psychoyios, and R. N. Markellos, "Modeling $\mathrm{CO}_{2}$ emission allowance prices and derivatives: evidence from the european trading scheme," Journal of Banking and Finance, vol. 33, pp. 1230-1241, 2009.

[36] M. Mansanet-Bataller, J. Chevallier, M. Hervé-Mignucci, and E. Alberola, "EUA and sCER phase II price drivers: unveiling the reasons for the existence of the EUA-sCER spread," Energy Policy, vol. 39, pp. 1056-1069, 2011.

[37] D. Rittler, "Price discovery and volatility spillovers in the European Union emissions trading scheme: a high-frequency analysis," Journal of Banking \& Finance, vol. 36, pp. 774-785, 2012.

[38] N. Koch, S. Fuss, G. Grosjean, and O. Edenhofer, "Causes of the EU ETS price drop: recession, CDM, renewable policies or a bit of everything?-new evidence," Energy Policy, vol. 73, pp. 676-685, 2014.

[39] Q. Tu and J. L. Mo, "Coordinating carbon pricing policy and renewable energy policy with a case study in China," Computers \& Industrial Engineering, vol. 113, pp. 294-304, 2017.

[40] K. R. French, G. W. Schwert, and R. F. Stambaugh, "Expected stock returns and volatility," Journal of Financial Economics, vol. 19, pp. 3-29, 1987.

[41] D. B. Nelson, "Conditional Heteroskedasticity in asset returns: a new approach," Econometrica, vol. 59, no. 2, pp. 347-370, 1991.

[42] J. Chevallier, "Modelling risk premia in $\mathrm{CO}_{2}$ allowances spot and futures prices," Economic Modelling, vol. 27, pp. 717-729, 2010.

[43] F. G. Kalantzis and N. T. Milonas, "Analyzing the impact of futures trading on spot price volatility: evidence from the spot electricity market in France and Germany," Energy Economics, vol. 36, pp. 454-463, 2013.

[44] G. S. Uddin, J. A. Hernandez, S. J. H. Shahzad, and A. Hedström, "Multivariate dependence and spillover effects across energy commodities and diversification potentials of carbon assets," Energy Economics, vol. 71, pp. 35-46, 2018.
[45] S. Viteva, Y. V. Veld-Merkoulova, and K. Campbell, "The forecasting accuracy of implied volatility from ECX carbon options," Energy Economics, vol. 45, pp. 475-484, 2014.

[46] J. Chevallier, "Detecting instability in the volatility of carbon prices," Energy Economics, vol. 33, no. 1, pp. 99-110, 2011.

[47] X. P. Tan and X. Y. Wang, "Dependence changes between the carbon price and its fundamentals: a quantile regression approach," Applied Energy, vol. 190, pp. 306-325, 2017.

[48] V. N. Vapnik, "An overview of statistical learning theory," IEEE Transactions on Neural Networks, vol. 10, no. 5, pp. 988-999, 1999.

[49] A. H. Alizadeh, C.-Y. Huang, and S. Van Dellen, "A regime switching approach for hedging tanker shipping freight rates," Energy Economics, vol. 49, pp. 44-59, 2015. 